\title{
Marcel J. Mélançon
}

Philosophe, professeur chercheur en bioéthique à l'Université du Québec à Chicoutimi

Directeur du Groupe de recherche en génétique et éthique du Québec (GÉNÉTHIQ)

(1984)

\section{"Le pacifisme médical face à la guerre nucléaire.”}

\begin{abstract}
Un document produit en version numérique par Jean-Marie Tremblay, bénévole, professeur de sociologie retraité du Cégep de Chicoutimi

Courriel: jean-marie tremblay@uqac.ca

Site web pédagogique : http://www.uqac.ca/jmt-sociologue/
\end{abstract}

Dans le cadre de: "Les classiques des sciences sociales" Une bibliothèque numérique fondée et dirigée par Jean-Marie Tremblay, professeur de sociologie au Cégep de Chicoutimi Site web: http://classiques.uqac.ca/

Une collection développée en collaboration avec la Bibliothèque Paul-Émile-Boulet de l'Université du Québec à Chicoutimi Site web: http://bibliotheque.uqac.ca/ 


\section{Politique d'utilisation de la bibliothèque des Classiques}

Toute reproduction et rediffusion de nos fichiers est interdite, même avec la mention de leur provenance, sans l'autorisation formelle, écrite, du fondateur des Classiques des sciences sociales, Jean-Marie Tremblay, sociologue.

Les fichiers des Classiques des sciences sociales ne peuvent sans autorisation formelle:

- être hébergés (en fichier ou page web, en totalité ou en partie) sur un serveur autre que celui des Classiques.

- servir de base de travail à un autre fichier modifié ensuite par tout autre moyen (couleur, police, mise en page, extraits, support, etc...),

Les fichiers (.html, .doc, .pdf, .rtf, .jpg, .gif) disponibles sur le site Les Classiques des sciences sociales sont la propriété des Classiques des sciences sociales, un organisme à but non lucratif composé exclusivement de bénévoles.

Ils sont disponibles pour une utilisation intellectuelle et personnelle et, en aucun cas, commerciale. Toute utilisation à des fins commerciales des fichiers sur ce site est strictement interdite et toute rediffusion est également strictement interdite.

L'accès à notre travail est libre et gratuit à tous les utilisateurs. C'est notre mission.

Jean-Marie Tremblay, sociologue

Fondateur et Président-directeur général, LES CLASSIQUES DES SCIENCES SOCIALES. 
Cette édition électronique a été réalisée par Jean-Marie Tremblay, bénévole, professeur de sociologie au Cégep de Chicoutimi à partir de :

\section{Marcel J. Mélançon}

Philosophe, professeur chercheur en bioéthique à l'Université du Québec à Chicoutimi Directeur du Groupe de recherche en génétique et éthique du Québec (GÉNÉTHIQ)

\section{“Le pacifisme médical face à la guerre nucléaire.”}

Un article publié dans la revue Considérations, revue de philosophie, vol. 6, no 3, 1984, pp. 61-67. Québec : Faculté de philosophie, Université Laval.

[Autorisation formelle accordée par l'auteur le 27 septembre 2008 de diffuser toutes ses publications dans Les Classiques des sciences sociales. Cette autorisation a été reconfirmée le 30 mars 2012.]

Courriel : marcel_melancon@uquac.ca

Polices de caractères utilisée :

Pour le texte: Times New Roman, 12 points.

Pour les citations : Times New Roman, 12 points.

Pour les notes de bas de page : Times New Roman, 10 points.

Édition électronique réalisée avec le traitement de textes Microsoft Word 2008 pour Macintosh.

Mise en page sur papier format : LETTRE US, 8.5'’ x 11’’

Édition numérique réalisée le 14 juin 2012 à Chicoutimi, Ville de Saguenay, Québec. 


\section{Marcel J. Mélançon}

Philosophe, professeur chercheur en bioéthique à l'Université du Québec à Chicoutimi Directeur du Groupe de recherche en génétique et éthique du Québec (GÉNÉTHIQ)

\section{"Le pacifisme médical face à la guerre nucléaire.”}

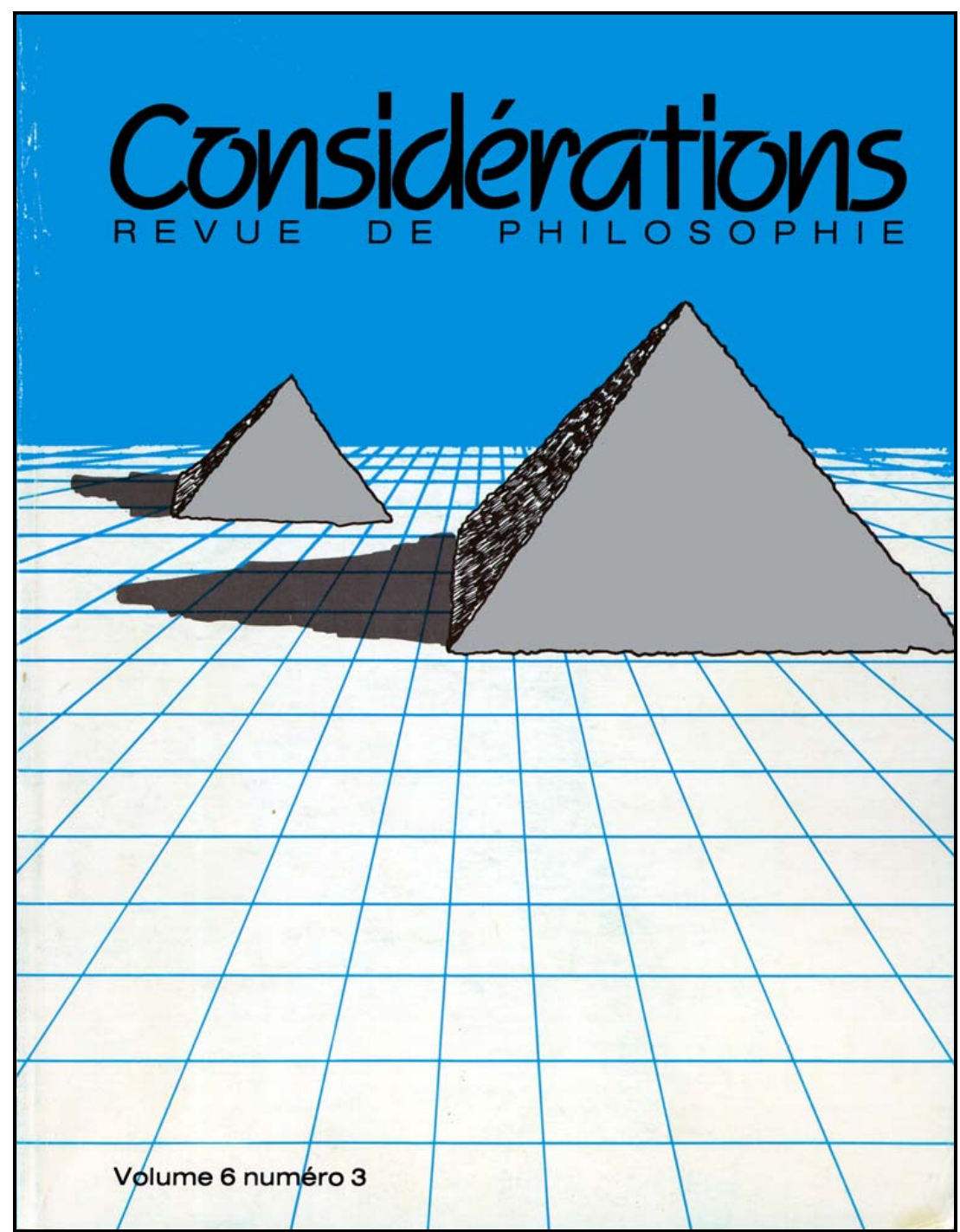

Un article publié dans la revue Considérations, revue de philosophie, vol. 6, no 3, 1984, pp. 61-67. Québec : Faculté de philosophie, Université Laval. 


\section{Table des matières}

$\underline{\text { Introduction }}$

I. Médecine, guerre et paix

II. Le mouvement pacifiste

\section{$\underline{\text { Conclusion }}$}




\section{Marcel J. Mélançon}

Philosophe, professeur chercheur en bioéthique à l'Université du Québec à Chicoutimi

Directeur du Groupe de recherche en génétique et éthique du Québec (GÉNÉTHIQ)

\section{“Le pacifisme médical face à la guerre nucléaire.”}

Un article publié dans la revue Considérations, revue de philosophie, vol. 6, no 3, 1984, pp. 61-67. Québec : Faculté de philosophie, Université Laval.

\section{Introduction}

Retour à la table des matières

Il existe un courant de pacifisme en médecine. Aux États-Unis, le corps médical mène, surtout depuis 1981, une action socio-politique pour informer sur les conséquences d'une guerre nucléaire sur la santé publique. L'American Medical Association et la Harvard Medical School se sont inscrites dans cette optique, de même que de grandes revues médicales ${ }^{1}$, telles le New England Journal of Medecine $^{2}$ et le JAMA ${ }^{3}$.

Le mouvement le plus organisé et le plus influent est le Physicians for Social Responsibility (PSR). Lorsqu'il sera question de "pacifisme médical" ici, c'est surtout à ce groupe qu'il sera fait référence.

Après quelques considérations sur la médecine, et la guerre, les principaux arguments pacifistes seront résumés et discutés brièvement.

1 Joyce BERMEL, "Should Physicians Prepare for War ?", The Hastings Center Report, Volume 12, Number 2, April 1982, p. 15.

2 Bernard LOWN, Eric CHIVIAN, James MULLER, Herbert ABRAMS, "The Nuclear-Arms Race and the Physician". The New England Journal of Medicine, Volume 304, Number 12, March 19, 1981, p. 726-729.

3 Bernard LOWN, "Physicians and Nuclear War", JAMA, Volume 246,, Number 20, November 20. 1981, p. 2331-2333. 


\section{Médecine, guerre et paix}

$\underline{\text { Retour à la table des matières }}$

De par ses principes et ses objectifs, la médecine est pacifique et, dans une certaine mesure pacifiste. "Guérir et, sinon guérir, du moins soulager, [62] mais en aucune manière nuire" ("primum non nocere") sont la raison d'être et l'objectif des corps médicaux : prévenir les causes de maladie (médecine préventive) pour les traiter (médecine curative) ou les soulager lorsqu'elle ne peut guérir (médecine palliative). Prendre soin de la santé physique ou mentale individuelle et collective, c'est faire oeuvre de paix et non de violence. Objectifs qui sont en contradiction même avec la guerre qui détruit, aliène ou handicape des vies humaines. Il serait aberrant, de ce point de vue, de poser une cause ou de militer en faveur d'une cause qui détériorerait la vie humaine.

En principe et par principe la médecine est pacifique, même si en pratique elle va à la guerre pour gérer de son mieux la catastrophe militaire chez les civils ou chez les combattants. "Pacifisme" n'a rien ici d'un désengagement concret en temps de conflit, désengagement qui conduirait à un "acharnement pacifiste" (il y a une distinction, en effet, entre l'acharnement pacifique et l'acharnement pacifiste). Elle accompagne les militaires des deux côtés des frontières et est active sur les champs de bataille. Deux figures ont particulièrement illustré le pacifisme médical : Florence Nightingale à la guerre de Crimée et Henri Dunant à la bataille de Solferino.

Les diverses conventions internationales, dont les Conventions de Genève (1864-1949), ont conféré un statut institutionnel, professionnel et international de neutralité aux corps médical,, Infirmier et hospitalier. Ils sont non-combattants. Ils se doivent de respecter, protéger et traiter les malades et les blessés qui ne peuvent être laissés sans assistance médicale. La différence de race, de sexe, de nationalité surtout, ne peut entrer en ligne de compte dans les traitements. La coercition physique ou psychologique, l'utilisation de l'art médical ne peuvent être employées pour des fins militaires ou para-militaires, notamment pour obtenir des informations. Bref. la médecine en temps de guerre s'adresse à des êtres humains et non à des combattants d'idéologie ou d'obédience différentes. 
Ni les conflits d'obligations ni l'obéissance à des "ordres supérieurs" 4 ne peuvent justifier qu'elle déroge à sa fonction humanitaire. Le tribunal de Nuremberg a condamné, en 1946, 26 médecins nazis qui avaient fait des expériences Irrecevables sur des prisonniers. L'art médical pourrait disposer d'un arsenal puissant, par exemple en pharmacologie et en psychiatrie, pour la torture en temps de guerre ou en temps de paix. Les sciences médicales et biomédicales ont des obligations sociales 5 et ne peuvent être vouées à des causes militaires ou para-militaires.

La médecine ne peut donc, sans trahir sa tradition historique, ses principes et objectifs. son mandat social et son statut international, participer à la guerre ni collaborer à la planification d'une guerre.

Peut-elle cependant participer à l'élaboration de plans de survie, de traitements et de soins, en prévision de l'éventualité d'une guerre nucléaire accidentelle ou provoquée ? C'est la question sur laquelle se sont penchés le Physicians for Social Responsibility (PSR) et ses sympathisants (médecins ou non).

\section{Le mouvement pacifiste}

$\underline{\text { Retour à la table des matières }}$

Formé en 1961, le PSR s'est donné comme objectif une action sociopolitique face à la guerre nucléaire : informer le public et le corps médical, influencer le gouvernement sur les conséquences médicales des expériences nucléaires et sur la guerre nucléaire elle-même. Ce groupe de 60 médecins de l'hôpital de Contra Costa (Californie) s'est surtout opposé en 1981 à une politique de collaboration entre le Département de la défense et l'Association des médecins et des hôpitaux. Quels sont les événements ? Quels sont les arguments de cette organisation et de ses sympathisants?

4 E.A. VASTYAN, "Medicine and War", The Encycloeedia of Bioethics, Warren T. REICH, Ed., New York : The Free Press, 1978, p. 1695-1699.

5 Victor W. SIDEL and Mark SIDEL, "Biomedical Science and War", The Encyclopedia of Bioethics, cit., p. 1699-1704. 
A. La guerre conventionnelle apparaît comme artisanale en comparaison de la guerre nucléaire. Les changements rapides dans le domaine de l'armement ont abouti depuis la IIe guerre mondiale comme on le sait, à l'arsenal sophistiqué des engins termo-nucléaires et des missiles tactiques de divers calibres et portées. La prolifération des armes nucléaires, l'échec plus ou moins perpétuel des pourparlers sur la limitation des armes stratégiques et sur le désarmement, l'escalade de la tension politique ont rendu de plus en plus probable le déclenchement accidentel ou provoqué d'un conflit nucléaire. À ce point qu'en 1981, le Département de la défense américaine envoyait une lettre aux hôpitaux civils du pays, leur demandant 50,000 lits pour les blessés qui seraient rapatriés par pont aérien des lieux d'un éventuel conflit outre-atlantique, les hôpitaux militaires ou de vétérans ne pouvant recevoir cet afflux imprévu. Le qualificatif de "nucléaire" n'était pas employé, mais le contexte et la description des soins laissaient croire que c'était le cas. 6

Ce plan, nécessitant la collaboration de la médecine civile et du Gouvernement face à l'éventualité d'une telle guerre, reçut l'accord de l'American Medical Association et de l'American Hospitals Association, notamment. Cependant le groupe de médecins du-15-ST S'objecta : la médecine ne peut participer à l'élaboration de plans de survie, de soins et traitements, d'évacuation des blessés militaires et d'hospitalisation dans les hôpitaux civils. Quels sont les arguments du PSR et de ses sympathisants ? Qu'en penser?

\section{B. Les arguments}

Premier argument : Une telle collaboration serait une approbation tacite d'une politique de préparation de guerre nucléaire - limitée ou totale.

Une analogie illustre cette proposition. Thomas Murray ${ }^{7}$ la propose. Supposons qu'une administration publique, incompétente ou fourbe, planifie la construction d'un système d'égouts inadéquat qui fait encourir des risques de diverses ma-

6 H. Jack GEIGER, "Why Survival Plans Are Meaningless", The Hastings Center Report, cit., p. 17-19.

7 Thomas H. MURRAY, "The Physician as Moral Leader", The Hastings Center Report, cit., p. 20-21. 
ladies ou d'épidémie. Supposons aussi que des médecins soient contactés pour collaborer à la préparation d'un plan de traitement dans le cas où les gens seraient atteints. Ces médecins devraient refuser pour deux raisons : cette collaboration rendrait le plan d'égout plus acceptable pour le public parce qu'il tient la médecine en haute estime, et cette collaboration violerait leur devoir envers les patients et la communauté en acquiesçant à une politique qui met leur santé en péril. Ce serait moralement inacceptable.

Trois choses sont à dire sur cette analogie. D'abord la comparaison est trop modeste, la préparation d'un système d'attaque nucléaire est infiniment plus dégo0tante. Ensuite la comparaison suppose que les maladies éventuelles seraient circonscrites localement. Enfin l'épidémie serait contrôlable et traitable. Mais l'analogie est valable en ceci qu'il y aurait une approbation médicale tacite.

Cet argument est le plus lourd dans l'arsenal pacifiste. Il obtiendrait toute sa force de frappe si la médecine ne contrebalançait pas cette collaboration par un autre type d'action : protestation publique des organisations médicales contre la préparation de guerre, déclaration officielle de dissidence d'avec une politique militariste, tenue de symposia, de cours, ou autres, pour lever toute ambigüité possible sur la position de la médecine.

Second argument : $\quad$ En cas de guerre nucléaire, tous les plans de survie seraient à ce point inefficaces que leur préparation est dénuée de toute signification. ${ }^{8}$

Cet argument repose sur la différence entre une guerre conventionnelle et la guerre nucléaire. Les 120,000 lits des hôpitaux militaires et de vétérans seraient débordés en 48 heures. Un estimé de 188.000 blessés transportables [65] dans les premiers jours d'un conflit nucléaire limité rendrait le pont aérien transatlantique totalement débordé. De plus, une guerre nucléaire limitée et outre-mer est une utopie. L'unicité du genre de destruction, son extension, les risques subis pour les humains et l'environnement par les engins thermonucléaires sont tels que toute planification de survie et de soins est absurde. Les études médicales en ce domaine le démontrent : tout effort médical serait inefficace. Un traitement de masse de

8 H. Jack GEIGER, ibid. 
milliers (voire de millions) de blessés est impossible à envisager pratiquement. Le scénario de l'ampleur des dégâts pour les survivants a été étudié par H. Abrams et W. Von Kaenel. 9

Trois observations peuvent être faites sur cet argument. Premièrement Il est Incontestable que les plans médicaux seraient sévèrement déficients et que la médecine en serait réduite à moins que de l'artisanat en cas de conflit nucléaire généralisé (stocks contaminés ou vite épuisés, hôpitaux pris d'assaut, médecins euxmêmes affectés, etc.). Cependant, qu'en serait-il si la médecine n'avait eu aucune préparation ? Le désastre humain serait pire encore et le lot de souffrance serait catastrophique. À l'absence d'espoir se joindrait le désespoir. Troisièmement, si des plans de survie sont prévus, la médecine pourrait encore, même d'une façon Infinitésimale, apporter des secours d'ultime détresse. Si elle n'était pas en mesure de donner des traitements curatifs, elle pourrait au moins assurer quelques soins palliatifs (ne fut-ce que pour quelques-uns).

Troisième argument : La participation médicale aux plans de survie augmente les risques de guerre nucléaire. ${ }^{10}$

Une guerre éventuelle serait déclenchée rapidement, et par des instances qui ne suivraient pas ou qui n'auraient pas l'opportunité de consulter le processus démocratique (vote du Congrès, opinion publique, etc.). De plus, l'engagement d'une société dans une guerre se fait pas à pas, et chaque pas est en apparence inoffensif. Ici, la médecine ferait faire un pas de plus si elle cautionnait le plan de survie. En outre l'engagement médical donnerait une fausse assurance : la guerre serait limitée, on pourrait y survivre, la médecine serait là pour traiter les blessés.

Cet argument, réfuté par J.C. Bisgard ${ }^{11}$, suppose que se préparer à recevoir des blessés rend la guerre plus probable. Mais le fait d'être bien [66] ou mal préparé médicalement n'a Jamais, historiquement parlant, prévenu ou retardé le dé-

9 Herbert L. ABRAMS, William E. VON KAENEL, "Medical Problems of Survivors of Nuclear War", The New England Journal of Medicine, Volume 305, Number 20, November 12, 1981, p. 1226-1232.

10 H. Jack GEIGER, ibid.

11 Jay C. BISGARD, "The Obligation to Care for Casualties", The Hastings Center Report, cit., p. 15-17. 
clenchement d'une guerre. Les leaders politiques qui ont calculé leurs chances de gagner une guerre n'ont pas été influencés par la situation de la médecine face aux blessés à soigner.

Deuxièmement, une saine prudence dicte qu'il faut se préparer dans l'éventualité de désastres naturels (épidémies, inondations, tremblements de terre) ou accidentels (chute d'avion, écroulement d'édifice). Cette préparation ne provoque pas ces catastrophes et nous serions mal venus de détruire les plans d'intervention ou de ne pas les élaborer, sous prétexte qu'on augmenterait ou diminuerait la possibilité de tels accidents. ${ }^{12}$ Dans le cas d'une calamité nucléaire. il en est de même : ce n'est pas la mise sur pied de plans de survie qui va, par elle-même, provoquer ou non l'événement. En d'autres termes, Il n'y a pas de lien de cause à effet entre se préparer à soigner des blessés de guerre et déclencher cette guerre.

Quatrième argument : Une guerre nucléaire serait nécessairement injuste.

Face aux effets d'une guerre nucléaire, la question historique de la guerre "juste" et "Injuste" a perdu toute signification morale. Elle doit être envisagée non plus en termes dichotomiques, mais dans un terme univoque : l'absurdité. Nécessairement Immorale, une guerre nucléaire planétaire serait la pire des Injustices que des politiciens absurdes Infligeraient à l'humanité. Traditionnellement, une guerre pouvait être un bienfait pour l'économie de pays non-alignés, fournisseurs des belligérants. Mats elle n'a jamais été pour le bien commun des pays concernés. Désormais la guerre nucléaire serait un désastre conjoint pour les protagonistes et une calamité universelle pour les non-belligérants. Des kilos d'atomes donneraient des mégatonnes de cadavres.

Deux choses, face à cet argument. Premièrement, les décisions de guerre sont hors de portée de la médecine. Deuxièmement, l'immoralité d'une guerre est une question indépendante de celle de la moralité face aux blessés de guerre. "Does it matter whether the war is just or not ? Emphatically, no. The Justice or injustice of a particular war has nothing at all to do with the obligation to care for the victims of that war, whether they be military or civilians, whether they be our own

12 Ibid. 
nationals or not... In the present connection. whatever particular critics of the CMCHS plan may think about the morality of war as such or about United States military preparedness in particular is not relevant to the question of contingency planning to care for wounded individuals". 13

\section{Conclusion}

Retour à la table des matières

Dans une civilisation qui se conduit encore en barbare (la guerre en est une manifestation, puisque nous ne pouvons pas régler nos conflits par la politique), une certaine "éthique par temps barbares" ou une "éthique dans la barbarie" est moralement justifiable. Elle tient compte des circonstances historiques. Elle peut soutenir qu'il est moralement acceptable, faute de mieux, de prévoir des plans de traitements en cas de catastrophe nucléaire.

L'engagement médical traditionnel pour la paix n'est pas incompatible avec un tel type d'action. Ce serait de l'“acharnement pacifiste” que de tenir le discours suivant: "Si une guerre nucléaire éclate, il appartiendra aux militaires de gérer leur propre catastrophe". Vouloir punir ainsi les auteurs de guerre serait une abdication de responsabilités envers les victimes de leur guerre.

Un engagement médical "prévisionnel" doit cependant assumer un autre type d'action socio-politique pour ne pas prêter le flanc (tant auprès du public que des politiciens) à l'accusation d'une approbation tacite d'une préparation militaire à une guerre nucléaire. La médecine devrait demander un budget spécial aux gouvernements pour un programme (tout aussi spécial) de traitement des politiciens qui ont un mal de guerre.

Refuser de se préparer médicalement à une guerre nucléaire serait prendre en otage, par anticipation, des milliers ou des millions de survivants qui auraient perdu en temps de guerre les soins auxquels ils ont droit en temps de paix.

13 James T. JOHNSON, "The Moral Bases of Contingency Planning", The Hastings Center Report, cit., p. 20. 
En terminant, ces quelques observations sur quelques arguments de médecins pacifistes ne concluent en rien quant à la raison d'être de leurs organisations. Au contraire, ils ont un r6le social important à jouer.

\section{Fin du texte}

